1 Hacettepe Journal of Mathematics and Statistics

$\bigcap$ Volume 44 (5) (2015), 991-1009

\title{
Orlicz-Lorentz spaces and their multiplication operators
}

\author{
René Erlin Castillo * , Héctor Camilo Chaparro ${ }^{\dagger}$ and Julio César Ramos \\ Fernández
}

\begin{abstract}
The boundedness, closed range, invertibility, compactness and closedness of multiplication operators on Orlicz-Lorentz spaces are characterized in this paper.
\end{abstract}

Keywords: Compact operator, Multiplication operator, Orlicz-Lorentz spaces. 2000 AMS Classification: primary 47B33, 47B38, secondary 46E30.

Received 05/11/2013 : Accepted 27/06/2014 Doi : 10.15672/HJMS.2015449663

\section{Introduction}

Let $f$ a complex-valued measurable function defined on a $\sigma$-finite measure space $(X, \mathcal{A}, \mu)$. For $\lambda \geq 0$, define $D_{f}(\lambda)$ the distribution function of $f$ as

$$
D_{f}(\lambda)=\mu(\{x \in X:|f(x)|>\lambda\}) .
$$

Observe that $D_{f}$ depends only on the absolute value $|f|$ of the function $f$ and $D_{f}$ may assume the value $+\infty$.

The distribution function $D_{f}$ provides information about the size of $f$ but not about the behavior of $f$ itself near any given point. For instance, a function on $\mathbb{R}^{n}$ and each of its translates have the same distribution function. It follows from (1.1) that $D_{f}$ is a decreasing function of $\lambda$ (not necessarily strictly) and continuous from the right.

Let $(X, \mu)$ be a measurable space and $f$ and $g$ be a measurable functions on $(X, \mu)$ then $D_{f}$ enjoy the following properties for all $\lambda_{1}, \lambda_{2} \geq 0$ :

(1) $|g| \leq|f| \mu$-a.e. implies that $D_{g} \leq D_{f}$;

(2) $D_{c f}(\lambda)=D_{f}\left(\frac{\lambda}{|c|}\right)$ for all $c \in \mathbb{C} \backslash\{0\}$;

*Universidad Nacional de Colombia, Departamento de Matemáticas, Bogotá, Colombia Email: recastillo@unal.edu.co

${ }^{\dagger}$ Universidad Nacional de Colombia, Departamento de Matemáticas, Bogotá, Colombia Email:hcchaparrog@unal.edu.co

${ }^{\ddagger}$ Universidad de Oriente, Departamento de Matemáticas, 6101 Cumaná, Estado Sucre, Venezuela

Email:jcramos@udo.edu.ve 
(3) $D_{f+g}\left(\lambda_{1}+\lambda_{2}\right) \leq D_{f}\left(\lambda_{1}\right)+D_{g}\left(\lambda_{2}\right)$;

(4) $D_{f g}\left(\lambda_{1} \lambda_{2}\right) \leq D_{f}\left(\lambda_{1}\right)+D_{g}\left(\lambda_{2}\right)$.

For more details on distribution function see [7].

By $f^{*}$ we mean the non-increasing rearrangement of $f$ given as

$$
f^{*}(t)=\inf \left\{\lambda>0: D_{f}(\lambda) \leq t\right\}, \quad t \geq 0
$$

where we use the convention that $\inf \emptyset=\infty . f^{*}$ is decreasing and right-continuous. Notice

$$
f^{*}(0)=\inf \left\{\lambda>0: D_{f}(\lambda) \leq 0\right\}=\|f\|_{\infty},
$$

since

$$
\|f\|_{\infty}=\inf \{\alpha \geq 0: \mu(\{x \in X:|f(x)|>\alpha\})=0\} .
$$

Also observe that if $D_{f}$ is strictly decreasing, then

$$
\left.f^{*}\left(D_{f}(t)\right)=\inf \left\{\lambda>0: D_{f}(\lambda) \leq D_{(} f\right) t\right\}=t .
$$

This fact demonstrates that $f^{*}$ is the inverse function of the distribution function $D_{f}$. Let $\mathcal{F}(X, \mathcal{A})$ denote the set of all $\mathcal{A}$-measurable functions on $X$. Let $\left(X, \mathcal{A}_{0}, \mu\right)$ and $\left(Y, \mathcal{A}_{1}, \nu\right)$ be two measure spaces.

Two functions $f \in F\left(X, \mathcal{A}_{0}\right)$ and $g \in F\left(X, \mathcal{A}_{1}\right)$ are said to be equimeasurable if they have the same distribution function, that is, if

$$
\mu(\{x \in X:|f(x)|>\lambda\})=\nu(\{y \in Y:|g(y)|>\lambda\}), \quad \text { for all } \lambda \geq 0 .
$$

So then there exists only one right-continuous decreasing function $f^{*}$ equimeasurable with $f$. Hence the decreasing rearrangement is unique.

In what follows, we gather some useful properties of the decreasing rearrangement function:

a) $f^{*}$ is decreasing.

b) $f^{*}(t)>\lambda$ if and only if $D_{f}(\lambda)>t$.

c) $f$ and $f^{*}$ are equimeasurables, that is

$$
D_{f}(\lambda)=D_{f^{*}}(\lambda) \quad \text { for all } \lambda \geq 0 .
$$

d) If $|f| \leq \liminf _{n \rightarrow \infty}\left|f_{n}\right|$ then $f^{*} \leq \liminf _{n \rightarrow \infty} f_{n}^{*}$.

e) If $E \in \mathcal{A}$, then $\left(\chi_{E}\right)^{*}(t)=\chi_{[0, \mu(E))}(t)$.

f) If $E \in \mathcal{A}$, then $\left(f \chi_{E}\right)^{*}(t) \leq f^{*}(t) \chi_{[0, \mu(E))}(t)$.

A weight is a nonnegative locally integrable function on $\mathbb{R}^{n}$ that takes values in $(0, \infty)$ almost everywhere. Therefore, weights are allowed to be zero or infinite only on a set of Lebesgue measure zero.

Let $\varphi:[0, \infty) \rightarrow[0, \infty)$ be a convex function such that

(1) $\varphi(x)=0$ if and only if $x=0$;

(2) $\lim _{x \rightarrow \infty} \varphi(x)=\infty$.

Such as function is known as a Young function. A Young function is strictly increasing, in fact, let $0<x<y$ then $0<\frac{x}{y}<1$ and hence, we might write

$$
x=\left(1-\frac{x}{y}\right) 0+\frac{x}{y} y .
$$


Since $\varphi$ is convex, we have

$$
\begin{aligned}
\varphi(x) & =\varphi\left(\left(1-\frac{x}{y}\right) 0+\frac{x}{y} y\right) \\
& \leq\left(1-\frac{x}{y}\right) \varphi(0)+\frac{x}{y} \varphi(y) \\
& <\varphi(y) .
\end{aligned}
$$

A Young function is said to satisfy the $\Delta_{2}$-condition if there exists a nonnegative constant $x_{0}$ and $k$ such that

$$
\varphi(2 x) \leq k \varphi(x) \text { for } x \geq x_{0} .
$$

If $x_{0}=0$, we say that $\varphi$ satisfy globally the $\Delta_{2}$-condition. The smaller constant $k$ which satisfy (1.3) is denoted by $k_{\Delta}$.

1.1. Claim. If $\varphi$ is a Young function such that satisfy the $\Delta_{2}$-condition, then for each $r \geq 0$ there exists a constant $k_{\Delta}(r)$ such that

$$
\varphi(r x) \leq k_{\Delta}(r) \varphi(x)
$$

for $x>0$ large enough.

Proof of the claim. If $r>0$, we can choose $n \in \mathbb{N}$ such that $r \leq 2^{n}$. Then we can applied (1.3) $n$-times and use the fact that $\varphi$ is increasing to obtain

$$
\varphi(r x) \leq \varphi\left(2^{n} x\right) \leq k^{n} \varphi(x),
$$

and hence we have (1.4).

1.2. Example. The function $\varphi_{1}(x)=\frac{x^{p}}{p}$ with $p>1$ is a Young function which satisfy globally the $\Delta_{2}$-condition with $k_{\Delta}=\frac{2^{p}}{p}$.

1.3. Example. The function $\varphi_{2}(t)=t^{p} \log (1+t)$ with $p \geq 1$ and $t \geq 0$ is a Young function which satisfy the $\Delta_{2}$-condition, indeed, since

$$
\lim _{t \rightarrow \infty} \frac{\varphi_{2}(2 t)}{\varphi_{2}(t)}=\lim _{t \rightarrow \infty} \frac{2^{p} t^{p} \log (1+2 t)}{t^{p} \log (1+t)}=2^{p-1} .
$$

Also, $\varphi_{2}$ satisfy globally the $\Delta_{2}$-condition.

In fact, since for each $t \geq 0$ we have $(1+t)^{2} \geq 1+2 t$, then

$$
\begin{aligned}
\varphi_{2}(2 t) & =2^{p} t^{p} \log (1+2 t) \\
& \leq 2^{p+1} t^{p} \log (1+2 t) \\
& \leq 2^{p+1} \varphi_{2}(2 t) .
\end{aligned}
$$

1.4. Lemma. A Young function $\varphi$ satisfy the $\Delta_{2}$-condition if and only if there exist constants $\lambda>1$ and $t_{0}>0$ such that

$$
\frac{t p(t)}{\varphi(t)}<\lambda
$$

for all $t \geq t_{0}$, where $p$ is the right derivate of $\varphi$.

Proof. Suppose that $\varphi$ satisfy the $\Delta_{2}$-condition, then there exists a constant $k>0$ such that

$$
k \varphi(t) \geq \varphi(2 t)=\int_{0}^{2 t} p(s) d s>\int_{t}^{2 t} p(s) d s
$$


for $t$ large enough, since $p$ is increasing, then we have

$$
\int_{t}^{2 t} p(s) d s>t p(t)
$$

hence, for $t$ large enough, we obtain

$$
\frac{t p(t)}{\varphi(t)} \leq k .
$$

Conversely, if

$$
\frac{t p(t)}{\varphi(t)}<\lambda
$$

for all $t \geq t_{0}$, then

$$
\int_{t}^{2 t} \frac{p(s)}{\varphi(s)} d s<\lambda \int_{t}^{2 t} \frac{d s}{s}=\lambda \log 2 .
$$

Since $p(s)=\varphi^{\prime}(s)$, we have

$$
\log \left(\frac{\varphi(2 t)}{\varphi(t)}\right)<\lambda \log 2
$$

which implies that

$$
\varphi(2 t)<2^{\lambda} \varphi(t) .
$$

The following result show us that the Young functions which satisfy the $\Delta_{2}$-condition have a cross rate less than the function $t^{p}$ for some $p>1$.

1.5. Theorem. If $\varphi$ is a Young function which satisfy the $\Delta_{2}$-condition, then there exist constants $\lambda>1$ and $C>0$ such that

$$
\varphi(t) \leq C t^{\lambda}
$$

for $t$ large enough.

Proof. By (1.4) we can write

$$
\int_{t_{0}}^{t} \frac{p(s)}{\varphi(s)} d s<\lambda \int_{t_{0}}^{t} \frac{d s}{s}
$$

where $t \geq t_{0}$. Then

$$
\log \left(\frac{\varphi(t)}{\varphi\left(t_{0}\right)}\right)<\lambda \log \left(\frac{t}{t_{0}}\right)
$$

therefore

$$
\varphi(t)<\frac{\varphi\left(t_{0}\right)}{t_{0}^{\lambda}} t^{\lambda}
$$

And the proof is complete.

1.6. Example. The following are Young functions:

(1) $\varphi(x)=\frac{|x|^{p}}{p}$ with $p>1$.

(2) $\varphi(x)=e^{|x|}-|x|-1$.

(3) $\varphi(x)=e^{|x|^{\delta}}-1$ with $\delta>1$.

(4) $\varphi(x)= \begin{cases}0, & \text { if } 0 \leq x \leq 1 \\ +\infty, & \text { otherwise. }\end{cases}$ 
Related with the Young function $\varphi$, we define, for $t \geq 0$ the complementary function of Young function as

$$
\psi(t)=\sup \{t s-\varphi(s): s \geq 0\} .
$$

1.7. Example. If $\varphi(t)=\frac{1}{p} t^{p}$ with $p>1$ and $t \geq 0$, then its complementary function is $\psi(t)=\frac{1}{q} t^{q}$ where $\frac{1}{p}+\frac{1}{q}=1$.

Indeed, by definition we have

$$
\psi(t)=\sup \left\{t s-\frac{1}{p} s^{p}: s \geq 0\right\},
$$

next, for $t>0$ fixed, we can consider the function

$$
g(s)=t s-\frac{1}{p} s^{p}, \quad \text { with } s \geq 0 .
$$

It is not hard to check that $g$ achieved its maximum at $s=t^{\frac{1}{p-1}}$ which is given by

$$
g\left(t^{\frac{1}{p-1}}\right)=\frac{1}{q} t^{q}
$$

Hence

$$
\psi(t)=\sup \left\{t s-\frac{1}{p} s^{p}: s \geq 0\right\}=\frac{1}{q} t^{q} .
$$

1.8. Proposition. If $\varphi$ is a Young function, then its complementary function $\psi$ is also a Young function.

Proof. It is clear that $\psi(0)=0$ if and only if $x=0$. Now, we just need to show that $\psi$ is a convex function. To this end, let us choose $t_{1}, t_{2} \in[0,+\infty)$ and $\lambda \in[0,1]$. Then, by definition of $\psi$ we have

$$
\psi\left(\lambda t_{1}+(1-\lambda) t_{2}\right)=\sup \left\{s\left(\lambda t_{1}+(1-\lambda) t_{2}\right)-\varphi(s): s \geq 0\right\} .
$$

On the other hand

$$
\lambda \psi\left(t_{1}\right)=\lambda \sup \left\{s t_{1}-\varphi(s): s \geq 0\right\} \geq \lambda\left(s t_{1}-\varphi(s)\right) \forall s \geq 0
$$

and

$$
(1-\lambda) \psi\left(t_{2}\right)=(1-\lambda) \sup \left\{s t_{2}-\varphi(s): s \geq 0\right\} \geq(1-\lambda)\left(s t_{2}-\varphi(s)\right) \forall s \geq 0 .
$$

From the last two inequalities, we have

$$
\begin{aligned}
s\left(\lambda t_{1}+(1-\lambda) t_{2}\right)-\varphi(s) & =\lambda\left(s t_{1}-\varphi(s)\right)+(1-\lambda)\left(s t_{2}-\varphi(s)\right) \\
& \leq \lambda \psi\left(t_{1}\right)+(1-\lambda) \psi\left(t_{2}\right)
\end{aligned}
$$

for all $s \geq 0$. Which means that $\lambda \psi\left(t_{1}\right)+(1-\lambda) \psi\left(t_{2}\right)$ is an upper bound of the set

$$
\left\{s\left(\lambda t_{1}+(1-\lambda) t_{2}\right)-\varphi(s): s \geq 0\right\}
$$

then

$$
\left.\psi\left(\lambda t_{1}+(1-\lambda) t_{2}\right)\right) \leq \psi\left(t_{1}\right)+(1-\lambda) \psi\left(t_{2}\right),
$$

and so $\psi$ is convex.

1.9. Theorem (Young's Inequality). Let $\psi$ be the complementary function of $\varphi$. Then

$$
t s \leq \varphi(s)+\psi(t)
$$

where $t, s \in[0,+\infty)$. 
Proof. Let $t, s \in[0,+\infty)$. Then

$$
\begin{aligned}
\psi(t) & =\sup \{s t-\varphi(s): s \geq 0\} \\
& \geq s t-\varphi(s) \quad \forall s \geq 0,
\end{aligned}
$$

then

$$
\psi(t)+\varphi(s) \geq s t
$$

and the proof is complete.

For more details on Young functions see [10].

\section{Weighted Lorentz-Orlicz Spaces}

The aim of this section is to present basic results about Lorentz-Orlicz spaces. We have tried to make the proofs as self-contained and synthetic as possible.

2.1. Definition (Luxemburg norm). Let $\varphi$ be a Young function. For any measurable function $f$ on $X$,

$$
\|f\|_{\varphi, w}=\inf \left\{\varepsilon>0: \int_{0}^{\infty} \varphi\left(\frac{f^{*}(t)}{\varepsilon}\right) w(t) d t \leq 1\right\} \in[0, \infty) .
$$

Where it is understood that $\inf (\emptyset)=+\infty$.

2.2. Remark. In this article, we will not always require that the Luxemburg norm actually be a norm. $\|\cdot\|_{\varphi, w}$ is indeed a quasinorm. A quasinorm is a functional that is like a norm except that it does only satisfy the triangle inequality with a constant $C \geq 1$, that is, $\|f+g\| \leq C(\|f\|+\|g\|)$ where $C \geq 1$.

2.3. Lemma. For any measurable function $f$ on $X,\|f\|_{\varphi, w}=0$ if and only if $f=0$ $\mu$-almost everywhere.

Proof. Clearly $\|f\|_{\varphi, w}=0$ if and only if $\int_{0}^{\infty} \varphi\left(\frac{f^{*}(t)}{\varepsilon}\right) w(t) d t \leq 1 \forall \varepsilon>0$. It follows that

$$
\begin{aligned}
\|f\|_{\varphi, w}=0 & \text { if and only if } \int_{0}^{\infty} \varphi\left(\alpha f^{*}(t)\right) w(t) d t=0 \forall \alpha>0 \\
& \text { if and only if } \varphi\left(\alpha f^{*}(t)\right) w(t)=0 \mu \text {-a.e. } \forall \alpha>0 \\
& \text { if and only if } f^{*}(t)=0 \mu \text {-a.e. } \\
& \text { if and only if } D_{f}(\lambda)=0 \mu-\text { a.e. } \\
& \text { if and only if } f=0 \mu \text {-a.e. }
\end{aligned}
$$

Identification of almost everywhere equal functions. As with $L_{p}$ spaces, one identifies the function which are $\mu$-almost everywhere equal. This means that one works with the equivalence classes of the equivalence relation defined by the $\mu$-almost everywhere equality. From now on, this will be done without further mention. Consequently, one write:

$$
\|f\|_{\varphi, w}=0 \text { if and only if } f=0 .
$$

2.4. Lemma. If $0<\|f\|_{\varphi, w}<\infty$ then $\int_{0}^{\infty} \varphi\left(\frac{f^{*}(t)}{\|f\|_{\varphi, w}}\right) w(t) d t \leq 1$. In particular, $\|f\|_{\varphi, w} \leq 1$ is equivalent to $\int_{0}^{\infty} \varphi\left(f^{*}(t)\right) w(t) d t \leq 1$. 
Proof. For all $b>\|f\|_{\varphi, w}$, we have

$$
\int_{0}^{\infty} \varphi\left(\frac{f^{*}(t)}{b}\right) w(t) d t \leq 1
$$

Letting $b$ decrease to $\|f\|_{\varphi, w}$, one obtains the first result by monotone convergence. The second statement follows from this and lemma 2.8.

2.5. Proposition. The gauge $\|\cdot\|_{\varphi, w}$ is a quasinorm on the vector space of all the measurable functions $f$ such that $\|f\|_{\varphi, w}<\infty$.

Proof. It is already seen that (2.1) holds under identification of a.e. equal functions.

It is clear that for all real $\lambda,\|\lambda f\|_{\varphi, w}=|\lambda|\|f\|_{\varphi, w}$.

It remains to prove the triangle inequality. Let $f$ and $g$ be two measurable functions such that $0<\|f\|_{\varphi, w}+\|g\|_{\varphi, w}<\infty$. Then

$$
\begin{aligned}
& \int_{0}^{\infty} \varphi\left(\frac{(f+g)^{*}(t)}{2\left(\|f\|_{\varphi, w}+\|g\|_{\varphi, w}\right)}\right) w(t) d t \\
\leq & \int_{0}^{\infty} \varphi\left(\frac{f^{*}(t / 2)+g^{*}(t / 2)}{2\left(\|f\|_{\varphi, w}+\|g\|_{\varphi, w}\right)}\right) w(t) d t \\
= & \int_{0}^{\infty} \varphi\left(\frac{\|f\|_{\varphi, w}}{2\left(\|f\|_{\varphi, w}+\|g\|_{\varphi, w}\right)} \frac{f^{*}(t / 2)}{\|f\|_{\varphi, w}}+\frac{\|g\|_{\varphi, w}}{2\left(\|f\|_{\varphi, w}+\|g\|_{\varphi, w}\right)} \frac{g^{*}(t / 2)}{\|g\|_{\varphi, w}}\right) w(t) d t \\
\leq & \frac{\|f\|_{\varphi, w}}{2\left(\|f\|_{\varphi, w}+\|g\|_{\varphi, w}\right)} \int_{0}^{\infty} \varphi\left(\frac{f^{*}(t / 2)}{\|f\|_{\varphi, w}}\right) w(t) d t \\
& +\frac{\|g\|_{\varphi, w}}{2\left(\|f\|_{\varphi, w}+\|g\|_{\varphi, w}\right)} \int_{0}^{\infty} \varphi\left(\frac{g^{*}(t / 2)}{\|f\|_{\varphi, w}}\right) w(t) d t \\
= & \frac{\|f\|_{\varphi, w}}{2\left(\|f\|_{\varphi, w}+\|g\|_{\varphi, w}\right)} 2 \int_{0}^{\infty} \varphi\left(\frac{f^{*}(t)}{\|f\|_{\varphi, w}}\right) w(2 t) d t \\
& +\frac{\|g\|_{\varphi, w}}{2\left(\|f\|_{\varphi, w}+\|g\|_{\varphi, w}\right)} 2 \int_{0}^{\infty} \varphi\left(\frac{g^{*}(t)}{\|f\|_{\varphi, w}}\right) w(2 t) d t \\
\leq & \frac{\|f\|_{\varphi, w}}{\|f\|_{\varphi, w}+\|g\|_{\varphi, w}} \int_{0}^{\infty} \varphi\left(\frac{f^{*}(t)}{\|f\|_{\varphi, w}}\right) w(t) d t \\
& +\frac{\|g\|_{\varphi, w}}{\|f\|_{\varphi, w}+\|g\|_{\varphi, w}} \int_{0}^{\infty} \varphi\left(\frac{g^{*}(t)}{\|f\|_{\varphi, w}}\right) w(t) d t \\
\leq & 1 .
\end{aligned}
$$

Where the last but one inequality follows from the convexity of $\varphi$ and the fact that $w$ is nonincreasing and the last inequality from lemma 2.4. Therefore

$$
\|f+g\|_{\varphi, w} \leq 2\left(\|f\|_{\varphi, w}+\|g\|_{\varphi, w}\right) .
$$

As a consequence, the set of all measurable functions $f$ such that $\|f\|_{\varphi, w}<\infty$ is a vector space.

2.6. Definition. Let $\varphi$ be a Young function. We define the weighted Lorenz-Orlicz spaces

$$
L_{\varphi, w}=\left\{f: X \rightarrow \mathbb{C} \text { measurable }: \int_{0}^{\infty} \varphi\left(\alpha f^{*}(t)\right) w(t) d t<\infty, \text { for some } \alpha>0\right\} .
$$

It follows from proposition 1.8 that if $L_{\varphi, w}$ is a weighted Lorentz-Orlicz space, then $L_{\psi, w}$ is also a weighted Lorenz-Orlicz space. 
2.7. Proposition (Hölder's type inequality). For $f \in L_{\varphi, 1}$ and $g \in L_{\psi, 1}$

$$
\int_{X}|f g| d \mu \leq 2\|f\|_{\varphi, 1}\|g\|_{\psi, 1} \text {. }
$$

In particular, $f g \in L_{1}$.

Proof. If $\|f\|_{\varphi, 1}=0$ or $\|g\|_{\psi, 1}=0$, one concludes with lemma 2.8 .

Assume now that $0<\|f\|_{\varphi, 1},\|g\|_{\psi, 1}$. Because of Young's inequality: $s t \leq \varphi(s)+\varphi(t)$ we have

$$
\begin{aligned}
\int_{X} \frac{|f g|}{\|f\|_{\varphi, 1}\|g\|_{\psi, 1}} d \mu & \leq \int_{0}^{\infty} \frac{f^{*}(t) g^{*}(t)}{\|f\|_{\varphi, 1}\|g\|_{\psi, 1}} d t \\
& \leq \int_{0}^{\infty} \varphi\left(\frac{f^{*}(t)}{\|f\|_{\varphi, 1}}\right) d t+\int_{0}^{\infty} \psi\left(\frac{g^{*}(t)}{\|g\|_{\psi, 1}}\right) d t \\
& \leq 2 .
\end{aligned}
$$

Therefore

$$
\int_{X}|f g| d \mu \leq 2\|f\|_{\varphi, 1}\|g\|_{\psi, 1}
$$

2.8. Lemma. Let $\left\{f_{n}\right\}_{n \in \mathbb{N}}$ be a sequence in $L_{\varphi, w}$. Then, the following assertions are equivalent:

(a) $\lim _{n \rightarrow \infty}\left\|f_{n}\right\|_{\varphi, w}=0$

(b) For all $\alpha>0$, $\limsup _{n \rightarrow \infty} \int_{0}^{\infty} \varphi\left(\alpha f_{n}^{*}(t)\right) w(t) d t \leq 1$;

(c) For all $\alpha>0, \lim _{n \rightarrow \infty} \int_{0}^{\infty} \varphi\left(\alpha f_{n}^{*}(t)\right) w(t) d t=0$.

Proof. The equivalence $(a) \Leftrightarrow(b)$ is a direct consequence of the definition of $\|\cdot\|_{\varphi, w}$. Off course $(c) \Rightarrow(b)$ is obvious. As $\varphi$ is convex and $\varphi(0)=0$ for all $t \geq 0$ and $0<\varepsilon \leq 1$, we have

$$
\varphi(t)=\varphi\left((1-\varepsilon) 0+\varepsilon \frac{t}{\varepsilon}\right) \leq(1-\varepsilon) \varphi(0)+\varepsilon \varphi\left(\frac{t}{\varepsilon}\right),
$$

that is

$$
\varphi(t) \leq \varepsilon \varphi\left(\frac{t}{\varepsilon}\right) \quad t \geq 0,0<\varepsilon \leq 1
$$

From which $(b) \Rightarrow(c)$ follows easily.

2.9. Theorem. The space $L_{\varphi, w}$ is a quasi-Banach space.

Proof. Let $\left\{f_{n}\right\}_{n \in \mathbb{N}}$ be a Cauchy sequence in $L_{\varphi, w}$. Let us choose $\tilde{\varepsilon}>0$ such that $\tilde{\varepsilon} \varphi^{-1}\left(\frac{\varepsilon}{k_{0}}\right)<\frac{1}{n+m}$ for $n, m \in \mathbb{N}$ and $\varepsilon>0, k_{0}>0$. For such $\tilde{\varepsilon}$ there exists $n_{0} \in \mathbb{N}$ such that

$$
\left\|f_{n}-f_{m}\right\|_{\varphi, w}<\tilde{\varepsilon}
$$

If $n, m \geq n_{0}$. By the definition of the Luxemburg quasi-norm we can use $k_{0}>0$ in such a way that $k_{0}<\tilde{\varepsilon}$ and

$$
\int_{0}^{\infty} \varphi\left(\frac{\left(f_{n}-f_{m}\right)^{*}(t)}{k_{0}}\right) w(t) d t \leq 1
$$

Let $E=\left\{x \in X:\left|f_{n}(x)-f_{m}(x)\right|>\varepsilon\right\}$, then

$$
\varepsilon \chi_{E}(x) \leq\left|f_{n}(x)-f_{m}(x)\right| \text {. }
$$


And hence

$$
\begin{aligned}
\varepsilon \chi_{E}^{*}(t) & \leq\left(f_{n}-f_{m}\right)^{*}(t), \\
\varepsilon \chi_{(0, \mu(E))}(t) & \leq\left(f_{n}-f_{m}\right)^{*}(t) .
\end{aligned}
$$

Therefore

$$
\int_{0}^{\infty} \varphi\left(\frac{\varepsilon}{k_{0}} \chi_{(0, \mu(E))}(t)\right) w(t) d t \leq \int_{0}^{\infty} \varphi\left(\frac{\left(f_{n}-f_{m}\right)^{*}(t)}{k_{0}}\right) w(t) d t .
$$

Then

$$
\begin{aligned}
& \int_{0}^{\mu(E)} \varphi\left(\frac{\varepsilon}{k_{0}}\right) w(t) d t \leq \int_{0}^{\infty} \varphi\left(\frac{\left(f_{n}-f_{m}\right)^{*}(t)}{k_{0}}\right) w(t) d t \\
\Rightarrow & \tilde{\varepsilon} \int_{0}^{D_{f_{n}-f_{m}(\varepsilon)}} w(t) d t \leq \tilde{\varepsilon} \varphi^{-1}\left(\frac{\varepsilon}{k_{0}}\right) \int_{0}^{\infty} \varphi\left(\frac{\left(f_{n}-f_{m}\right)^{*}(t)}{k_{0}}\right) w(t) d t \\
\Rightarrow & \tilde{\varepsilon} \int_{0}^{D_{f_{n}-f_{m}(\varepsilon)}} w(t) d t \leq \frac{1}{n+m} \\
\Rightarrow & \tilde{\varepsilon} \lim _{n, m \rightarrow \infty} \int_{0}^{D_{f_{n}-f_{m}(\varepsilon)} w(t)=0 .}
\end{aligned}
$$

Since $w>0$, we must have $\lim _{n, m \rightarrow \infty} D_{f_{n}-f_{m}}(\varepsilon)=0$ which means that $\left\{f_{n}\right\}_{n \in \mathbb{N}}$ is a Cauchy sequence in measure, then some subsequence $\left\{f_{n_{k}}\right\}_{k \in \mathbb{N}}$ converges almost everywhere to a measurable function $f$, that is, $f_{n_{k}} \rightarrow f \mu$-a.e.

Let $\alpha>0$. By lemma 2.8 there exists a large enough integer $n(\alpha)$ such that

$$
\int_{0}^{\infty} \varphi\left(\alpha\left(f_{n}-f_{m}\right)^{*}(t)\right) w(t) d t \leq 1, \quad \forall m, n \geq n(\alpha)
$$

With Fatou's lemma this gives

$$
\int_{0}^{\infty} \varphi\left(\alpha\left(f_{n}-f\right)^{*}(t)\right) w(t) d t \leq \liminf \int_{0}^{\infty} \varphi\left(\alpha\left(f_{n}-f_{m}\right)^{*}(t)\right) w(t) d t \leq 1
$$

$\forall m \geq n(\alpha)$. Therefore $f_{n}-f$ belongs to $L_{\varphi, w}$, but $f_{n} \in L_{\varphi, w}$, so that $f \in L_{\varphi, w}$.

Moreover, as $\limsup _{m \rightarrow \infty} \int_{0}^{\infty} \varphi\left(\alpha\left(f_{m}-f\right)^{*}(t)\right) w(t) d t \leq 1$ for all $\alpha>0$, we have $\lim _{m \rightarrow \infty}\left\|f_{m}-f\right\|_{\varphi, w}=0$. This proves that $L_{\varphi, w}$ is complete.

2.10. Theorem. Simple functions are dense in $L_{\varphi, w}$.

Proof. Suppose $f \in L_{\varphi, w}$. We may assume that $f \geq 0$. Note that if $D_{f}(\lambda)=\infty$, then $\lim _{t \rightarrow \infty} f^{*}(t)=0$. It follows that $D_{f}(\lambda)<\infty$.

Hence, given $\varepsilon, \delta>0$, we can find a simple function $s_{n} \geq 0$ such that $s_{n}(x)=0$ when $f(x) \leq \varepsilon$ and $f(x)-\varepsilon \leq s_{n}(x) \leq f(x)$ when $f(x)>\varepsilon$ except on a set of measure less than $\delta$. It follows that

$$
\mu\left(\left\{x \in X:\left|f(x)-s_{n}(x)\right|>\varepsilon\right\}\right)<\delta .
$$

Next, choose $n \in \mathbb{N}$ such that $n \geq \frac{1}{\varepsilon}$, then

$$
\left(f-s_{n}\right)^{*}(t)=\inf \left\{\varepsilon>0: D_{f-s_{n}}(\varepsilon)<\delta \leq t\right\} .
$$

Thus

$$
\left(f-s_{n}\right)^{*}(t) \leq \frac{1}{n} \quad \text { for } t \geq \delta,
$$

since $s_{n} \leq f$, then $s_{n}^{*}(t) \leq f^{*}(t)$, for each $t>0$. Since $n>\frac{1}{\varepsilon}$, we have

$$
\left(f-s_{n}\right)^{*}(t) \leq \frac{1}{n}<\varepsilon
$$


next,

$$
\int_{0}^{\infty} \varphi\left(\frac{\left(f-s_{n}\right)^{*}(t)}{k}\right) w(t) d t \leq \int_{0}^{\infty} \varphi\left(\frac{1}{n k}\right) w(t) d t .
$$

Let $a=\int_{0}^{\infty} w(t) d t$, then

$$
\begin{aligned}
\left\|f-s_{n}\right\|_{\varphi, w} & =\inf \left\{k>0: \int_{0}^{\infty} \varphi\left(\frac{\left(f-s_{n}\right)^{*}(t)}{k}\right) w(t) d t \leq 1\right\} \\
& =\frac{1}{n \varphi^{-1}\left(\frac{1}{a}\right)} \rightarrow 0 \quad \text { as } n \rightarrow \infty .
\end{aligned}
$$

\section{Multiplication Operator}

Let $F(X)$ be a function space on non-empty set $X$. Let $u: X \rightarrow \mathbb{C}$ be a function such that $u \cdot f \in F(X)$ whenever $f \in F(X)$.

Then, the transformation $f \mapsto u \cdot f$ on $F$ is denoted by $M_{u}$. In case $F(X)$ is a topological space and $M_{u}$ is continuos, we call it a multiplication operator induced by $u$.

Multiplication operators generalize the notion of operator given by a diagonal matrix. More precisely, one of the results of operator theory is a spectral theorem, which states that every self-adjoint operator on a Hilbert space is unitarily equivalent to a multiplication operator on an $L_{2}$ space.

These operators received considerable attention over the past several decades specially on $L_{p}$ spaces and Bergman spaces and they played an important role in the study of operators on Hilbert spaces.

For more details on these operators we refer to Abrahamese [1], Axler [4], Douglas [6], Halmos [8] and Takagi [12].

3.1. Example. Consider the Hilbert space $X=L_{2}[-1,3]$ of complex-valued square integrable functions on the interval $[-1,3]$. Define the operator

$$
M_{u}(x)=u(x) x^{2},
$$

for any function $u \in X$. This will be a self-adjoint bounded linear operator with norm 9. Its spectrum will be the interval [0,9] (the range of the function $x \rightarrow x^{2}$ defined on $[-1,3])$. Indeed, for any complex number $\lambda$, the operator $M_{u}-\lambda$ is given by

$$
\left(M_{u}-\lambda\right)(x)=u(x)\left(x^{2}-\lambda\right) .
$$

It is invertible if and only if $\lambda$ is not in $[0,9]$, and then its inverse is

$$
\left(M_{u}-\lambda\right)^{-1}(x)=\frac{u(x)}{x^{2}-\lambda} .
$$

which is another multiplication operator.

For a systematic study of the multiplication operators on different spaces we refer to $[1,3,4,5,9,11]$.

3.2. Remark. In general, the multiplication operators on measurable spaces is not $1-1$. Indeed, let $(X, \mathcal{A}, \mu)$ be a measure space and

$$
A=X \backslash \operatorname{supp}(u)=\{x \in X: u(x)=0\} .
$$

If $\mu(A) \neq 0$ and $f=\chi_{A}$ then for any $x \in X$ we have $f(x) u(x)=0$ which implies that $M_{u}(f)=0$, therefore $\operatorname{ker}\left(M_{u}\right) \neq\{0\}$ and hence $M_{u}$ is not $1-1$.

If, on the contrary, $M_{u}$ is $1-1$, then $\mu(X \backslash \operatorname{supp}(u))=0$. On the other hand, if $\mu(X \backslash \operatorname{supp}(u))=0$ and $\mu$ is a complete measure, then $M_{u}(f)=0$ implies $f(x) u(x)=$ $0 \forall x \in X$, then $\{x \in X: f(x) \neq 0\} \subseteq X \backslash \operatorname{supp}(u)$ and so $f=0 \mu$-a.e. on $X$.

Hence, if $\mu(X \backslash \operatorname{supp}(u))=0$ and $\mu$ is a complete measure, then $M_{u}$ is $1-1$. 
3.3. Proposition. $M_{u}$ is $1-1$ on $Y=L_{\varphi, w}(\operatorname{supp} u)$.

Proof. Let $Y=L_{\varphi, w}(\operatorname{supp} u)=\left\{f \chi_{\operatorname{supp} u}: f \in L_{\varphi, w}\right\}$. Indeed, if $M_{u}(\tilde{f})=0$ with $\tilde{f}=f \chi_{\operatorname{supp} u} \in Y$, then $f(x) \chi_{\operatorname{supp} u}(x) u(x)=0$ for all $x \in X$, and so

$$
\begin{aligned}
& f(x) u(x)=0 \quad \forall x \in \operatorname{supp}(u), \\
\Rightarrow & f(x)=0 \quad \forall x \in \operatorname{supp}(u), \\
\Rightarrow & f(x) \chi_{\operatorname{supp} u(x)}(x)=0 \quad \forall x \in X .
\end{aligned}
$$

Then $\tilde{f}=0$ and the proof is complete.

In what follows, boundedness and invertibility of the multiplication $M_{u}$ are characterized in terms of the boundedness and invertibility of the complex valued measurable function $u$ respectively.

3.4. Theorem. The linear transformation $M_{u}: f \rightarrow u \cdot f$ on the Orlicz-Lorentz space $L_{\varphi, w}$ is bounded if and only if $u$ is essentially bounded. Moreover

$$
\left\|M_{u}\right\|=\|u\|_{\infty} \text {. }
$$

Proof. Let $u \in L_{\infty}(\mu)$, note $|(u f)(x)| \leq\|u\|_{\infty}|f(x)|$, thus

$$
\begin{aligned}
\{x:|(u f)(x)|>\lambda\} & \subseteq\left\{x:\|u\|_{\infty}|f(x)|>\lambda\right\} \\
& =\left\{x:|f(x)|>\frac{\lambda}{\|u\|_{\infty}}\right\}
\end{aligned}
$$

then

$$
D_{u f}(\lambda) \leq D_{f}\left(\frac{\lambda}{\|u\|_{\infty}}\right)
$$

and so

$$
\left\{\lambda>0: D_{f}\left(\frac{\lambda}{\|u\|_{\infty}}\right) \leq t\right\} \subseteq\left\{\lambda>0: D_{u f}(\lambda) \leq t\right\}
$$

From this we have

$$
\begin{aligned}
\inf \left\{\lambda>0: D_{u f}(\lambda) \leq t\right\} & \leq \inf \left\{\lambda>0: D_{f}\left(\frac{\lambda}{\|u\|_{\infty}}\right) \leq t\right\} \\
& \leq \inf \left\{\alpha\|u\|_{\infty}>0: D_{f}(\alpha) \leq t\right\} \\
& =\|u\|_{\infty} \inf \left\{\alpha>0: D_{f}(\alpha) \leq t\right\} .
\end{aligned}
$$

Hence

$$
(u f)^{*}(t) \leq\|u\|_{\infty} f^{*}(t)
$$

Then

$$
\begin{aligned}
\int_{0}^{\infty} \varphi\left(\frac{(u f)^{*}(t)}{\|u\|_{\infty}\|f\|_{\varphi, w}}\right) w(t) d t & \leq \int_{0}^{\infty} \varphi\left(\frac{\|u\|_{\infty} f^{*}(t)}{\|u\|_{\infty}\|f\|_{\varphi, w}}\right) w(t) d t \\
& =\int_{0}^{\infty} \varphi\left(\frac{f^{*}(t)}{\|f\|_{\varphi, w}}\right) w(t) d t \leq 1
\end{aligned}
$$

Hence $f \in L_{\varphi, w}$ and

$$
\left\|M_{u} f\right\|_{\varphi, w} \leq\|u\|_{\infty}\|f\|_{\varphi, w} .
$$

Conversely, suppose $M_{u}$ is a bounded operator. If $u$ is not essentially bounded function, then for every $n \in \mathbb{N}$, the set $E_{n}=\{x \in X:|u(x)|>n\}$ has a positive measure. Now, we know that

$$
\chi_{E_{n}}^{*}(t)=\chi_{0, \mu\left(E_{n}\right)}(t)
$$


and note

$$
\left\{x: n \chi_{E_{n}}(x)>\lambda\right\} \subseteq\left\{x:\left|u \chi_{E_{n}}(x)\right|>\lambda\right\},
$$

then

$$
D_{n \chi_{E_{n}}}(\lambda) \leq D_{u \chi_{E_{n}}}(\lambda),
$$

from this we have

$$
\left\{\lambda>0: D_{u \chi_{E_{n}}}(\lambda) \leq t\right\} \subseteq\left\{\lambda>0: D_{n \chi_{E_{n}}}(\lambda) \leq t\right\} .
$$

Hence

$$
\inf \left\{\lambda>0: D_{n \chi_{E_{n}}}(\lambda) \leq t\right\} \leq \inf \left\{\lambda>0: D_{u \chi_{E_{n}}}(\lambda) \leq t\right\} .
$$

That is,

$$
\left(u \chi_{E_{n}}\right)^{*}(t) \geq n\left(\chi_{E_{n}}\right)^{*}(t) .
$$

This gives us

$$
\begin{aligned}
1 & \geq \int_{0}^{\infty} \varphi\left(\frac{\left(u \chi_{E_{n}}\right)^{*}(t)}{k}\right) w(t) d t \\
& \geq \int_{0}^{\infty} \varphi\left(\frac{\left(n \chi_{E_{n}}\right)^{*}(t)}{k}\right) w(t) d t,
\end{aligned}
$$

and so

$$
\begin{aligned}
\left\{k>0: \int_{0}^{\infty} \varphi\left(\frac{\left(u \chi_{E_{n}}\right)^{*}(t)}{k}\right)\right. & w(t) d t \leq 1\} \\
& \subseteq\left\{k>0: \int_{0}^{\infty} \varphi\left(\frac{\left(n \chi_{E_{n}}\right)^{*}(t)}{k}\right) w(t) d t \leq 1\right\},
\end{aligned}
$$

thus

$$
\begin{aligned}
\inf \left\{k>0: \int_{0}^{\infty} \varphi\left(\frac{\left(n \chi_{E_{n}}\right)^{*}(t)}{k}\right) w(t) d t \leq 1\right\} \leq \\
\inf \left\{k>0: \int_{0}^{\infty} \varphi\left(\frac{\left(u \chi_{E_{n}}\right)^{*}(t)}{k}\right) w(t) d t \leq 1\right\},
\end{aligned}
$$

which means that

$$
\left\|M_{u} \chi_{E_{n}}\right\|_{\varphi, w} \geq n\left\|\chi_{E_{n}}\right\|_{\varphi, w},
$$

this contradicts the boundedness of $M_{u}$. Hence $u$ must be essentially bounded.

Next, clearly by (3.1) we obtain

(3.2) $\quad\left\|M_{u}\right\| \leq\|u\|_{\infty}$.

For $\varepsilon>0$, let $E=\left\{x \in X:|u(x)| \geq\|u\|_{\infty}-\varepsilon\right\}$ (observe that $\mu(E)>0$ ), then

$$
\left\{x \in X:\left(\|u\|_{\infty}-\varepsilon\right) \chi_{E}(x)>\lambda\right\} \subseteq\left\{x \in X:\left|u \chi_{E}(x)\right|>\lambda\right\},
$$

then

$$
D_{\left(\|u\|_{\infty}-\varepsilon\right) \chi_{E}}(\lambda) \leq D_{u \chi_{E}}(\lambda)
$$

and so

$$
\left\{\lambda>0: D_{u \chi_{E}}(\lambda) \leq t\right\} \subseteq\left\{\lambda>0: D_{\left(\|u\|_{\infty}-\varepsilon\right) \chi_{E}} \leq t\right\}
$$

from this we have

$$
\inf \left\{\lambda>0: D_{\left(\|u\|_{\infty}-\varepsilon\right) \chi_{E}} \leq t\right\} \leq \inf \left\{\lambda>0: D_{u \chi_{E}}(\lambda) \leq t\right\} .
$$

Therefore

$$
\left(u \chi_{E}\right)^{*}(t) \geq\left(\|u\|_{\infty}-\varepsilon\right)\left(\chi_{E}\right)^{*}(t),
$$


then

$$
\int_{0}^{\infty} \varphi\left(\frac{\left(\|u\|_{\infty}-\varepsilon\right)\left(\chi_{E}\right)^{*}(t)}{\left\|M_{u} \chi_{E}\right\|_{\varphi, w}}\right) w(t) d t \leq \int_{0}^{\infty} \varphi\left(\frac{\left(u \chi_{E}\right)^{*}(t)}{\left\|M_{u} \chi_{E}\right\|_{\varphi, w}}\right) w(t) d t \leq 1,
$$

which implies that

$$
\left\|\left(\|u\|_{\infty}-\varepsilon\right) \chi_{E}\right\|_{\varphi, w} \leq\left\|M_{u} \chi_{E}\right\|_{\varphi, w},
$$

and

$$
\left(\|u\|_{\infty}-\varepsilon\right)\left\|\chi_{E}\right\|_{\varphi, w} \leq\left\|M_{u} \chi_{E}\right\|_{\varphi, w}
$$

hence

$$
\|u\|_{\infty}-\varepsilon \leq \frac{\left\|M_{u} \chi_{E}\right\|_{\varphi, w}}{\left\|\chi_{E}\right\|_{\varphi, w}}
$$

which provide that

$$
\left\|M_{u}\right\| \geq\|u\|_{\infty}-\varepsilon \quad \forall \varepsilon>0
$$

and so

$$
\left\|M_{u}\right\| \geq\|u\|_{\infty}
$$

Therefore

$$
\left\|M_{u}\right\|=\|u\|_{\infty} .
$$

We will need the following well known result.

3.5. Theorem. Let $T \in B(X, Y)$ where $X$ and $Y$ are Banach spaces. Then $T$ is bounded below if and only if $T$ is $1-1$ and has closed range.

For the proof of theorem 3.5 see [2].

3.6. Corollary. $M_{u}: L_{\varphi, w}(\operatorname{supp} u) \rightarrow L_{\varphi, w}(\operatorname{supp} u)$ has closed range if and only if $M_{u}$ is bounded below on $L_{\varphi, w}(\operatorname{supp} u)$.

This result is clear since $M_{u}$ is $1-1$ on $L_{\varphi, w}(\operatorname{supp} u)$. Moreover, if $u \neq 0 \mu$-a.e. on $X$ with $\mu$ a complete measure, then we have the following result.

3.7. Corollary. If $\mu \neq 0 \mu$-a.e. on $X$ and $\mu$ is a complete measure, then

$$
M_{u}: L_{\varphi, w}(X, \mathcal{A}, u) \rightarrow L_{\varphi, w}(X, \mathcal{A}, u)
$$

has a closed range if and only if $M_{u}$ is bounded below on $L_{\varphi, w}(X, \mathcal{A}, u)$.

3.8. Theorem. $M_{u}: L_{\varphi, w}(\operatorname{supp} u) \rightarrow L_{\varphi, w}(\operatorname{supp} u)$ has a closed range if and only if there exists $\delta>0$ such that $|u(x)|>\delta \mu$-a.e. on $\operatorname{supp} \mu$.

Proof. If there exists a $\delta>0$ such that $|u(x)| \geq \delta \mu$-a.e. on $\operatorname{supp}(u)$, then for $f \in L_{\varphi, w}$ and $t>0$ we have

$$
\left\{x:\left|\delta f \chi_{\operatorname{supp}(u)}(x)\right|>\lambda\right\} \subseteq\left\{x:\left|u f \chi_{\operatorname{supp}(u)}(x)\right|>\lambda\right\},
$$

and so

$$
D_{\delta f \chi_{\operatorname{supp}(u)}}(\lambda) \leq D_{u f \chi_{\operatorname{supp}(u)}}(\lambda),
$$

then

$$
\left\{\lambda>0: D_{u f \chi_{\operatorname{supp}(u)}}(\lambda) \leq t\right\} \subseteq\left\{\lambda>0: D_{\delta f \chi_{\operatorname{supp}(u)}}(\lambda) \leq t\right\},
$$

from this we have

$$
\inf \left\{\lambda>0: D_{\delta f \chi_{\operatorname{supp}(u)}}(\lambda) \leq t\right\} \leq \inf \left\{\lambda>0: D_{u f \chi_{\operatorname{supp}(u)}}(\lambda) \leq t\right\},
$$


thus

$$
\left(u f \chi_{\operatorname{supp}(u)}\right)^{*}(t) \geq \delta f \chi_{\operatorname{supp}(u)}^{*}(t),
$$

then we shall note that

$$
\begin{aligned}
\left\{k>0: \int_{0}^{\infty} \varphi\left(\frac{\left(u f \chi_{\operatorname{supp}(u)}\right)^{*}(t)}{k}\right) w(t) d t \leq 1\right\} \subseteq \\
\left\{k>0: \int_{0}^{\infty} \varphi\left(\frac{\left(\delta f \chi_{\operatorname{supp}(u)}\right)^{*}(t)}{k}\right) w(t) d t \leq 1\right\} .
\end{aligned}
$$

Hence

$$
\begin{aligned}
\inf \left\{k>0: \int_{0}^{\infty} \varphi\left(\frac{\left(\delta f \chi_{\operatorname{supp}(u)}\right)^{*}(t)}{k}\right) w(t) d t \leq 1\right\} \leq \\
\quad \inf \left\{k>0: \int_{0}^{\infty} \varphi\left(\frac{\left(u f \chi_{\operatorname{supp}(u)}\right)^{*}(t)}{k}\right) w(t) d t \leq 1\right\},
\end{aligned}
$$

which means that

$$
\left\|\delta f \chi_{\operatorname{supp}(u)}\right\|_{\varphi, w} \leq\left\|M_{u} f \chi_{\operatorname{supp}(u)}\right\|_{\varphi, w},
$$

thus

$$
\left\|M_{u} f \chi_{\operatorname{supp}(u)}\right\|_{\varphi, w} \geq \delta\left\|f \chi_{\operatorname{supp}(u)}\right\|_{\varphi, w} .
$$

Therefore $M_{u}$ has closed range.

Conversely, assume that $M_{u}$ has closed range on $L_{\varphi, w}(\operatorname{supp}(u))$. Since $M_{u}: L_{\varphi, w}(\operatorname{supp}(u)) \rightarrow$ $L_{\varphi, w}(\operatorname{supp}(u))$ is $1-1$, then $M_{u}$ is bounded below, then there exists an $\varepsilon>0$ such that

$$
\left\|M_{u} f\right\|_{\varphi, w} \geq \varepsilon\|f\|_{\varphi, w}
$$

for all $f \in L_{\varphi, w}(\operatorname{supp}(u))$. Let $E=\{x \in \operatorname{supp}(u):|u(x)|<\varepsilon / 2\}$.

If $\mu(E)>0$, then we can find a measurable set $F \subseteq E$ such that $\chi_{F} \in L_{\varphi, w}(\operatorname{supp}(u))$. Then

$$
\left\{x:\left|u \chi_{F}\right|>\lambda\right\} \subseteq\left\{x:\left|\frac{\varepsilon}{2} \chi_{F}\right|>\lambda\right\}
$$

and so

$$
D_{u \chi_{F}}(\lambda) \leq D_{\frac{\varepsilon}{2} \chi_{F}}(\lambda),
$$

from this we have

$$
\left\{\lambda>0: D_{\frac{\varepsilon}{2} \chi_{F}}(\lambda) \leq t\right\} \subseteq\left\{\lambda>0: D_{u \chi_{F}}(\lambda) \leq t\right\},
$$

then

$$
\inf \left\{\lambda>0: D_{u \chi_{F}}(\lambda) \leq t\right\} \leq \inf \left\{\lambda>0: D_{\frac{\varepsilon}{2} \chi_{F}}(\lambda) \leq t\right\}
$$

that is,

$$
\left(u \chi_{F}\right)^{*}(t) \leq\left(\frac{\varepsilon}{2} \chi_{F}\right)^{*}(t),
$$

and so

$$
\left(u \chi_{F}\right)^{*}(t) \leq \frac{\varepsilon}{2}\left(\chi_{F}\right)^{*}(t) .
$$


Therefore

$$
\begin{aligned}
\left\|M_{u} \chi_{F}\right\|_{\varphi, w} & =\inf \left\{\varepsilon>0: \int_{0}^{\infty} \varphi\left(\frac{\left(u \chi_{F}\right)^{*}(t)}{\varepsilon}\right) w(t) d t \leq 1\right\} \\
& \leq \inf \left\{\varepsilon>0: \int_{0}^{\infty} \varphi\left(\frac{\frac{\varepsilon}{2}\left(\chi_{F}\right)^{*}(t)}{\varepsilon}\right) w(t) d t \leq 1\right\} \\
& =\frac{\varepsilon}{2}\left\|\chi_{F}\right\|_{\varphi, w},
\end{aligned}
$$

which is a contradiction. Therefore $\mu(E)=0$. This completes the proof.

3.9. Corollary. If $\mu \neq 0 \mu$-a.e. on $X$, and $\mu$ is a complete measure, then $M_{u}$ has a closed range on $L_{\varphi, w}(X, \mathcal{A}, \mu)$ if and only if there exists $\delta>0$ such that $|u(x)| \geq \delta \mu$-a.e. on $X$.

Proof. The result follows as a consequence of

$$
L_{\varphi, w}(X, \mathcal{A}, \mu)=L_{\varphi, w}(\operatorname{supp} u)
$$

3.10. Theorem. The set of all multiplication operators on $L_{\varphi, w}$ is a maximal abelian subalgebra of the set $B\left(L_{\varphi, w}\right)$, the algebra of all bounded linear operators on $L_{\varphi, w}$.

Proof. Let

$$
\mathcal{H}=\left\{M_{u}: u \in L_{\infty}\right\}
$$

and consider the operator product

$$
M_{u} \cdot M_{v}=M_{u v}
$$

where $M_{u}, M_{v} \in \mathcal{H}$. Let us check that it is a Banach algebra. Let $u, v \in L_{\infty}$, then $|u| \leq\|u\|_{\infty}$ and $|v| \leq\|v\|_{\infty}$, therefore

$$
\|u v\|_{\infty} \leq\|u\|_{\infty}\|v\|_{\infty}
$$

this implies that the product is an inner operation, moreover the usual function product is associative, commutative and distributive respect to the sum and the scalar product, thus we conclude that $\mathcal{H}$ is a subalgebra of $B\left(L_{\varphi, w}\right)$. Now, we like to check that it is a maximal subalgebra, that is, given $N \in B\left(L_{\varphi, w}\right)$, if $N$ commute with $\mathcal{H}$, we have to prove that $N \in \mathcal{H}$. Consider the unit function $e: X \rightarrow \mathbb{C}$ defined by $e(x)=1$ for all $x \in X$. Let $N \in B\left(L_{\varphi, w}\right)$ be an operator which commute with $\mathcal{H}$ and let $\chi_{E}$ the characteristic function of a measurable set $E$. Then

$$
\begin{aligned}
N\left(\chi_{E}\right) & =N\left[M_{\chi_{E}}(e)\right] \\
& =M_{\chi_{E}}[N(e)] \\
& =\chi_{E} \cdot N(e) \\
& =N(e) \cdot \chi_{E} \\
& =M_{w} \cdot \chi_{E},
\end{aligned}
$$

where $w=N(e)$. Similarly

$$
N(s)=M_{w}(s)
$$

for any simple function.

Now, let us check that $w \in L_{\infty}$. By way of contradiction, assume that $w \notin L_{\infty}$, then the set

$$
E_{n}=\{x \in X:|w(x)|>n\}
$$

has a positive measure for each $n \in \mathbb{N}$. Note that

$$
M_{w}\left(\chi_{E_{n}}\right)(x)=w \chi_{E_{n}}(x) \geq n \chi_{E_{n}}(x)
$$


for all $x \in X$. By the monotonicity property of the distribution function we have

$$
D_{w \chi_{E_{n}}}(\lambda) \geq D_{\chi_{E_{n}}}\left(\frac{\lambda}{n}\right)
$$

From this

$$
\left\{\lambda>0: D_{w \chi_{E_{n}}}(\lambda) \leq t\right\} \subseteq\left\{\lambda>0: D_{\chi_{E_{n}}}\left(\frac{\lambda}{n}\right) \leq t\right\}
$$

Then

$$
\inf \left\{\lambda>0: D_{\chi_{E_{n}}}\left(\frac{\lambda}{n}\right) \leq t\right\} \leq \inf \left\{\lambda>0: D_{w \chi_{E_{n}}}(\lambda) \leq t\right\}
$$

Putting $\alpha=\frac{\lambda}{n}$, we have

$$
\left\|w \chi_{E_{n}}\right\|_{\varphi, w} \geq n\left\|\chi_{E_{n}}\right\|_{\varphi, w}
$$

since $\chi_{E}$ is a simple function, then by (3.3) we have

$$
M_{w}\left(\chi_{E_{n}}\right)=N\left(\chi_{E_{n}}\right) \text {. }
$$

Hence

$$
\left\|N\left(\chi_{E_{n}}\right)\right\|_{\varphi, w} \geq n\left\|\chi_{E_{n}}\right\|_{\varphi, w} .
$$

Therefore $N$ is an unbounded operator. This is a contradiction to the fact that $N$ is bounded.

So then $w \in L_{\infty}$ and by theorem $3.4 M_{w}$ is bounded.

Next, given $f \in L_{\varphi, w}$, there exists a nondecreasing sequence $\left\{s_{n}\right\}_{n \in \mathbb{N}}$ of measurable simple functions such that $\lim _{n \rightarrow \infty} s_{n}=f$, then by (3.3) we have

$$
\begin{aligned}
N(f) & =N\left(\lim s_{n}\right) \\
& =\lim N\left(s_{n}\right) \\
& =\lim M_{w}\left(s_{n}\right) \\
& =M_{w}\left(\lim s_{n}\right) \\
& =M_{w}(f) .
\end{aligned}
$$

Therefore $N(f)=M_{w}(f)$ for all $f \in L_{\varphi, w}$ and thus we conclude that $N \in \mathcal{H}$.

3.11. Corollary. The multiplication operator is invertible on $B\left(L_{\varphi, w}\right)$ if and only if is invertible on $L_{\infty}$.

Proof. Let $M_{u}$ be invertible. Then there exists $N \in B\left(L_{\varphi, w}\right)$ such that

$$
M_{u} \cdot N=N \cdot M_{u}=I
$$

where $I$ represent the identity operator. Let us check that $N$ commute with $\mathcal{H}$.

Let $M_{w} \in \mathcal{H}$, then

$$
M_{w} \cdot M_{u}=M_{u} \cdot M_{w} .
$$

Applying $N$ to (3.5) and by (3.4) we obtain

$$
\begin{aligned}
N \cdot M_{w} \cdot M_{u} \cdot N & =N \cdot M_{u} \cdot M_{w} \cdot N, \\
N \cdot M_{w} \cdot I & =I \cdot M_{w} \cdot N, \\
N \cdot M_{w} & =M_{w} \cdot N,
\end{aligned}
$$

and thus we conclude that $N$ commute with $\mathcal{H}$. By theorem $3.10 N \in \mathcal{H}$, then there exists $g \in L_{\infty}$ such that $N=M_{g}$, hence

$$
M_{u} \cdot M_{g}=M_{g} \cdot M_{u}=I,
$$

this implies that $u g=g u=1 \mu$-a.e., which means that $u$ is invertible on $L_{\infty}$. 
On the other hand, assume $u$ is invertible on $L_{\infty}$, that is, $\frac{1}{u} \in L_{\infty}$, then

$$
\begin{aligned}
M_{u} \cdot M_{\frac{1}{u}} & =M_{\frac{1}{u}} \cdot M_{u} \\
& =M_{\left(\frac{1}{u}\right) u} \\
& =M_{1}=I,
\end{aligned}
$$

which means that $M_{u}$ is invertible on $B\left(L_{\varphi, w}\right)$.

For the sake of completeness and the convenience of the reader, we gives here one definition and one lemma which will play an important role on the coming results.

3.12. Definition. Let $T$ be an operator. A subspace $V$ of $X$ is said to be invariant under $T$ (or simply $T$-invariant) whenever

$$
T(V) \subseteq V .
$$

3.13. Lemma. Let $T: X \rightarrow X$ be an operator. If $T$ is compact and $M$ is a closed $T$-invariant subspace of $X$, then $\left.T\right|_{M}$ is compact.

Proof. Let $\left\{x_{n}\right\}_{n \in \mathbb{N}}$ be a subsequence in $M \subseteq X$. Then $\left\{x_{n}\right\}_{n \in \mathbb{N}} \subseteq X$, thus there exists a subsequence $\left\{x_{n_{k}}\right\}_{k \in \mathbb{N}}$ of $\left\{x_{n}\right\}_{n \in \mathbb{N}}$ such that $T\left(x_{n_{k}}\right)$ converges in $X$, but $T\left(x_{n_{k}}\right) \subseteq T(M)$ since $\left\{x_{n_{k}}\right\}_{k \in \mathbb{N}} \subseteq M$. Then $T\left(x_{n_{k}}\right)$ converges on $\overline{T(M)} \subseteq \bar{M}=M$. Therefore $T\left(x_{n_{k}}\right)$ converges on $M$, hence $\left.T\right|_{M}$ is compact.

3.14. Theorem. Let $M_{u}$ be a compact operator. For $\varepsilon>0$ define

$$
A_{\varepsilon}(u)=\{x \in X:|u(x)| \geq \varepsilon\},
$$

and

$$
L_{\varphi, w}\left(A_{\varepsilon}(u)\right)=\left\{f \chi_{A_{\varepsilon}(u)}: f \in L_{\varphi, w}\right\} .
$$

Then $L_{\varphi, w}\left(A_{\varepsilon}(u)\right)$ is a closed invariant subspace of $L_{\varphi, w}$ under $M_{u}$. Moreover

$$
\left.M_{u}\right|_{L_{\varphi, w}\left(A_{\varepsilon}(u)\right)}
$$

is a compact operator.

Proof. Let $h, s \in L_{\varphi, w}\left(A_{\varepsilon}(u)\right)$ and $\alpha, \beta \in \mathbb{R}$. Then $h=f \chi_{A_{\varepsilon}(u)}$ and $s=g \chi_{A_{\varepsilon}(u)}$ where $f, g \in L_{\varphi, w}$ thus

$$
\begin{aligned}
\alpha h+\beta s & =\alpha\left(f \chi_{A_{\varepsilon}(u)}\right)+\beta\left(g \chi_{A_{\varepsilon}(u)}\right) \\
& =(\alpha f+\beta g) \chi_{A_{\varepsilon}(u)} \in L_{\varphi, w}\left(A_{\varepsilon}(u)\right),
\end{aligned}
$$

which means that $L_{\varphi, w}\left(A_{\varepsilon}(u)\right)$ is a subspace of $L_{\varphi, w}$.

Next, for all $h \in L_{\varphi, w}\left(A_{\varepsilon}(u)\right)$ we have

$$
\begin{aligned}
M_{u} h & =u h \\
& =u\left(f \chi_{A_{\varepsilon}(u)}\right) \\
& =(u f) \chi_{A_{\varepsilon}(u)},
\end{aligned}
$$

where $u f \in L_{\varphi, w}$. Therefore $M_{u} \in L_{\varphi, w}\left(A_{\varepsilon}(u)\right)$, which means that $L_{\varphi, w}\left(A_{\varepsilon}(u)\right)$ is an invariant subspace of $L_{\varphi, w}$ under $M_{u}$.

Now, let us show that $L_{\varphi, w}\left(A_{\varepsilon}(u)\right)$ is a closed set. Indeed, let $g$ a function belonging to the closure of $L_{\varphi, w}\left(A_{\varepsilon}(u)\right)$ then there exists a sequence $\left\{g_{n}\right\}_{n \in \mathbb{N}}$ in $L_{\varphi, w}\left(A_{\varepsilon}(u)\right)$ such that

$$
g_{n} \rightarrow g \text { in } L_{\varphi, w}
$$

Just remain to exhibit that $g$ belongs to $L_{\varphi, w}\left(A_{\varepsilon}(u)\right)$. Note that

$$
g=g \chi_{A_{\varepsilon}(u)}+g \chi_{A_{\varepsilon}^{c}(u)} \text {. }
$$


Next, we want to show that $g \chi_{A_{\varepsilon}^{c}(u)}=0$. In fact, given $\varepsilon_{1}>0$ there exists $n_{0} \in \mathbb{N}$ such that

$$
\begin{aligned}
\left\|g \chi_{A_{\varepsilon}^{c}(u)}\right\|_{\varphi, w} & =\left\|\left(g-g_{n_{0}}+g_{n_{0}}\right) \chi_{A_{\varepsilon}^{c}(u)}\right\|_{\varphi, w} \\
& =\left\|\left(g-g_{n_{0}}\right) \chi_{A_{\varepsilon}^{c}(u)}\right\|_{\varphi, w} \\
& \leq\left\|g-g_{n_{0}}\right\|_{\varphi, w}<\varepsilon_{1} .
\end{aligned}
$$

Thus, $g \chi_{A_{\varepsilon}^{c}(u)}=0$ which means that $g=g \chi_{A_{\varepsilon}(u)}$, that is, $g \in L_{\varphi, w}\left(A_{\varepsilon}(u)\right)$. Finally by lemma 3.13 we have

$$
\left.M_{u}\right|_{L_{\varphi, w}\left(A_{\varepsilon}(u)\right)}
$$

is a compact operator. And the proof is now complete.

3.15. Theorem. Let $M_{u} \in B\left(L_{\varphi, w}\right)$. Then $M_{u}$ is compact if and only if $L_{\varphi, w}\left(A_{\varepsilon}(u)\right)$ is finite dimensional for each $\varepsilon>0$.

Proof. If $|u(x)| \geq \varepsilon$, we should note that

$$
\left|u f \chi_{A_{\varepsilon}}(x)\right| \geq \varepsilon f \chi_{A_{\varepsilon}(u)}(x)
$$

and so

$$
\left\{x: \varepsilon f \chi_{A_{\varepsilon}(u)}(x)>\lambda\right\} \subseteq\left\{x:\left|u f \chi_{A_{\varepsilon}(u)}(x)\right|>\lambda\right\},
$$

thus

$$
D_{\varepsilon f \chi_{A_{\varepsilon}(u)}}(\lambda) \leq D_{u f \chi_{A_{\varepsilon}(u)}}(\lambda)
$$

then

$$
\left\{\lambda>0: D_{u f \chi_{A_{\varepsilon}(u)}}(\lambda) \leq t\right\} \subseteq\left\{\lambda>0: D_{\varepsilon f \chi_{A_{\varepsilon}(u)}}(\lambda) \leq t\right\}
$$

from this we have

$$
\inf \left\{\lambda>0: D_{\varepsilon f \chi_{A_{\varepsilon}(u)}}(\lambda) \leq t\right\} \leq \inf \left\{\lambda>0: D_{u f \chi_{A_{\varepsilon}(u)}}(\lambda) \leq t\right\}
$$

that is

$$
\left(\varepsilon f \chi_{A_{\varepsilon}(u)}\right)^{*}(t) \leq\left(u f \chi_{A_{\varepsilon}(u)}\right)^{*}(t) .
$$

Hence

$$
\begin{aligned}
& \left\{k>0: \int_{0}^{\infty} \varphi\left(\frac{\left(u f \chi_{A_{\varepsilon}(u)}\right)^{*}(t)}{k}\right) w(t) d t \leq 1\right\} \subseteq \\
& \left\{k>0: \int_{0}^{\infty} \varphi\left(\frac{\varepsilon\left(f \chi_{A_{\varepsilon}(u)}\right)^{*}(t)}{k}\right) w(t) d t \leq 1\right\} .
\end{aligned}
$$

Therefore

$$
\begin{aligned}
\inf \left\{k>0: \int_{0}^{\infty} \varphi\left(\frac{\varepsilon\left(f \chi_{A_{\varepsilon}(u)}\right)^{*}(t)}{k}\right) w(t) d t \leq 1\right\} \leq \\
\quad \inf \left\{k>0: \int_{0}^{\infty} \varphi\left(\frac{\left(u f \chi_{A_{\varepsilon}(u)}\right)^{*}(t)}{k}\right) w(t) d t \leq 1\right\} .
\end{aligned}
$$

And hence

$$
\left\|M_{u} f \chi_{A_{\varepsilon}(u)}\right\|_{\varphi, w} \geq \varepsilon\left\|f \chi_{A_{\varepsilon}(u)}\right\|_{\varphi, w} .
$$

Now, if $M_{u}$ is a compact operator, then $L_{\varphi, w}\left(A_{\varepsilon}(u)\right)$ is a closed invariant subspace of $L_{\varphi, w}$ under $M_{u}$ and by lemma 3.13

$$
\left.M_{u}\right|_{L_{\varphi, w}\left(A_{\varepsilon}(u)\right)}
$$


is a compact operator. Then by (3.6) $\left.M_{u}\right|_{L_{\varphi, w}\left(A_{\varepsilon}(u)\right)}$ has a closed range in $L_{\varphi, w}\left(A_{\varepsilon}(u)\right)$ and it is invertible, being compact $L_{\varphi, w}\left(A_{\varepsilon}(u)\right)$ is finite dimensional.

Conversely, suppose that $L_{\varphi, w}\left(A_{\varepsilon}(u)\right)$ is finite dimensional for each $\varepsilon>0$. In particular, for each $n, L_{\varphi, w}\left(A_{\frac{1}{u}}(u)\right)$ is finite dimensional, then for each $n$, define $u_{n}: X \rightarrow \mathbb{C}$ as

$$
u_{n}(x)= \begin{cases}u(x) & \text { if }|u(x)| \geq \frac{1}{n} \\ 0 & \text { if }|u(x)|<\frac{1}{n}\end{cases}
$$

Then we find that

$$
\left(\left(u_{n}-u\right) \cdot f\right)^{*}(t) \leq\left\|u_{n}-u\right\|_{\infty} f^{*}(t) \forall t>0 .
$$

Consequently

$$
\begin{aligned}
\left\|M_{u_{n}} f-M_{u} f\right\|_{\varphi, w} & \leq\left\|u_{n}-u\right\|_{\infty}\|f\|_{\varphi, w} \\
& \leq \frac{1}{n}\|f\|_{\varphi, w},
\end{aligned}
$$

which implies that $M_{u_{n}}$ converges to $M_{u}$ uniformly. As $L_{\varphi, w}\left(A_{\varepsilon}(u)\right)$ is finite dimensional so $M_{u_{n}}$ is a finite rank operator. Therefore, $M_{u_{n}}$ is a compact operator and hence $M_{u}$ is a compact operator.

\section{References}

[1] M.B. Abrahamese, Multiplication operators, Lecture notes in Math., Vol. 693 (1978), 17-36, Springer Verlag, New York, 1978.

[2] Y.A. Abramovich, C.D. Aliprantis, An Invitation to Operator Theory, American Mathematical Society, (2002).

[3] S.C. Arora, Gopal Datt and Satish Verma, Multiplication operators on lorentz spaces, Indian Journal of Mathematics, Vol 48 (3) (2006), 317-329.

[4] A. Axler, Multiplication operators on Bergman space, J. Peine Angew Math., Vol. 33 (6) (1982), 26-44.

[5] R.E. Castillo, R. León; E. Trousselot. Multiplication operator on $L(p, q)$ spaces, Panamer. Math Journal Vol 19 (2009) No. 1, 37-44.

[6] R.G. Douglas, Banach Algebra Techniques in Operator Theory, Academic Press, New York, 1972.

[7] Grafakos, Loukas. Classical Fourier Analysis Second Edition, Springer, (2008).

[8] P.R. Halmos, A Hilbert Space Problem Book, Van Nostrand, Princeton, N. J., 1961.

[9] B.S. Komal and Shally Gupta, Multiplication operators between Orlicz spaces, Integral Equations and Operator Theory. Vol. 41 (2001), 324-330.

[10] M.M. Rao and Z.D. Ren, Theory of Orlicz spaces, Marcel Dekker Inc., New York, 1993.

[11] R.K. Singh and A. Kumar, Multiplication and composition operators with closed ranges, Bull. Aust. Math. Soc. Vol. 16 (1977), 247-252.

[12] H. Takagi, Fredholm Weighted Composition Operators, Integral equations and Operator Theory 16 (1993), 267-276. 
